\title{
An apparatus for the assessment of prepulse inhibition in the frog
}

\author{
HOWARD S. HOFFMAN and FRANCIA RUPPEN \\ Bryn Mawr College, Bryn Mawr, Pennsylvania
}

\begin{abstract}
A system for studying prepulse inhibition in the leopard frog is described herein. It entails a modification of an apparatus developed almost a century ago, by Yerkes, for his investigations of hearing in the frog. With the present system, a frog is restrained in a cylindrical vessel (constructed from a 35-mm film canister), and the canister is positioned in the apparatus so that the animal's legs hang downward. A miniature solenoid is used to deliver a startle eliciting tap to the frog's head, and the resulting leg movement is detected by an optical densitometer. In addition to providing a convenient way to restrain frogs, our system enabled us to, in part, replicate Yerkes's basic procedures with these subjects. As did Yerkes, we found that an acoustic signal just prior to a tap on a frog's head inhibits the leg movement that would otherwise be elicited.
\end{abstract}

It is now just 10 years short of a century since Robert Yerkes published his classic monograph entitled "The Sense of Hearing in Frogs" (Yerkes, 1905). In it, Yerkes reported that while he was unable to detect any overt reaction of a frog in direct response to an acoustic signal, the frog's leg moved in response to a mild tap to the head, and, moreover, the amount of this movement could be systematically modified by a variety of appropriately presented acoustic signals. In particular, he found that the simultaneous ringing of a bell induced larger leg movement in response to the tap, whereas the leg movement was inhibited if the bell was sounded at an appropriate interval (approximately $500 \mathrm{msec}$ ) prior to the tap.

As noted by Ison and Hoffman (1983), Yerkes's procedure was the earliest American study in which prepulse inhibition was employed to assess sensory function in a lower animal. While prepulse inhibition has subsequently been the subject of numerous investigations (see Hoffman \& Ison, 1980, for a review), we have identified only two reports, beyond Yerkes's original studies, that assessed prepulse inhibition in frogs, and neither of these used the Yerkes leg flexion apparatus (Moss \& Simmons, 1986; Simmons, 1988). In the present report, we describe a series of modifications of Yerkes's original leg flexion apparatus that enhance both its ease of use and its objectivity. In doing so, they can extend Yerkes's methodology to a variety of sensory modalities besides audition and to a variety of new experimental issues concerning the nature of prepulse inhibition in the frog.

Yerkes (1905) described his method as follows: "A frog is placed in a saddle-like holder and kept in position with linen bands over the back and a wire screen cap over the head." He went on to note that "under these conditions the hind legs usually hang free and limp and any move-

Correspondence should be addressed to H. S. Hoffman, Department of Psychology, Dalton Hall, Bryn Mawr College, Bryn Mawr, PA 19010 (e-mail: hhoffman@cc.brynmawr.edu). ment which may be made by them in response to a stimulus can be read in millimeters from a scale attached to a holder" (p. 288).

About 3 years ago, we set out to try to replicate some of Yerkes's work. It seemed, at the time, that despite the paucity of prior studies, the frog ought to prove an easily housed and inexpensive subject for a detailed analysis of prepulse inhibition. We were also aware, however, that about 50 years earlier, Helen Peak had been unsuccessful when she attempted to replicate some of Yerkes's original research. In a 1982 letter to James Ison, Ernest Hilgard had commented: "I recall how hard Helen Peak worked to repeat Yerkes' work with the frog, in which the main problem was to find a holder out of which the frog couldn't escape." We had searched the literature for a report of that work, and since we discovered none, we assumed that Peak never found a satisfactory solution to this problem. Despite these forebodings of failure, we felt that the goal would be worth the effort. As might be expected, however, our initial efforts to properly secure our frogs also failed dismally. The slippery animals kept wriggling out of the linen bands with which we were trying to hold them.

Figure 1 shows the system that we eventually devised to provide a restraint from which our frogs would be unable to escape. Instead of trying to bind an animal directly to the apparatus, we constructed an independent holder from a $35-\mathrm{mm}$ plastic film canister of the sort that is available in most camera shops. Shown at the left in Figure 1, the canister was modified by cutting a corner off of its bottom end and smoothing the cut edges by using a miniature blowtorch to form a narrow bead. This provided a crescentshaped hole in the canister bottom that would be just large enough for a medium-sized leopard frog's head to pass through but would be too small to admit the rest of its body. The canister was also modified by cutting two small slots in its open end, as can be seen at the left in Figure 1. In addition, small pieces of hook Velcro were glued to the top and bottom of the canister, and a partially split length of 

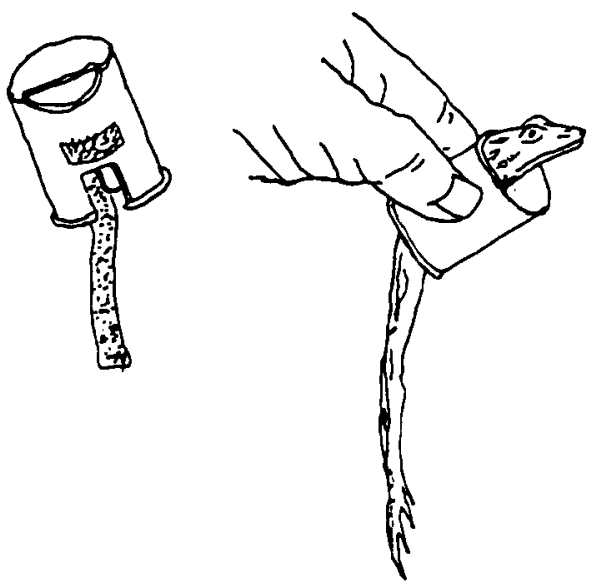

Figure 1. A modified 35-mm film canister is used to restrain a frog. The left side of this figure shows the manner in which the canister is modified (see the text for details). The right side of this figure shows a frog restrained within the canister.

loop Velcro was attached to the piece on the bottom. The purpose of the split is to accommodate the frog's ischium bone when the frog has been restrained in the holder.

The right side of Figure 1 shows a frog in the holder. To insert a frog, it is only necessary to grasp the animal's extended hind legs with one hand, and, while holding the canister with the other hand, place the animal's head in its open end. Usually, the frog will immediately attempt to escape by sticking its head through the crescent-shaped hole. When it does so, it can be secured within the canister by drawing the hanging piece of loop Velcro upward between the frog's legs and securing it to the hook Velcro on the top of the canister. Once restrained in the canister, a frog will usually remain perfectly still with its legs dangling, for hours.

Figure 2 shows the apparatus that we developed to carry out our tests. It is modeled after the leg flexion apparatus that Yerkes showed on page 290 of his monograph. A pair of wing-like extensions have been added to the saddle arrangement to provide a secure seat for the canister containing the frog. In addition, a moveable arm is provided to carry the device used to deliver taps. With these exceptions, our apparatus is very similar to the one that Yerkes employed.

The body of Figure 2 shows our apparatus with its arm in its raised position so as to facilitate the placement of a canister containing a frog at the start of a test session. When the canister has been properly positioned, a strip of loop Velcro is passed over it and is tightly fastened to strips of hook Velcro that have been permanently glued to the underside of the wings. Next, the arm is lowered until it rests against a stop, at which point it will be above and parallel to the canister. The inset in Figure 2 shows this arrangement and shows the way in which taps are delivered. Whereas Yerkes used the fall of a miniature mallet to deliver a tap, we employ a miniature solenoid (Guardian Electric TB $3.5 \times 924 \mathrm{~V} \mathrm{dc}$ ) for this purpose. The solenoid is fitted with a coiled internal spring so as to return the plunger to its initial position after each tap delivery. A 4-mm-diameter Teflon cone with a rounded end is permanently attached to the distal end of the plunger. Once a frog is in place and the support arm lowered, the exact placement of the solenoid is adjusted until the tip of the cone is about $4 \mathrm{~mm}$ above the midline of the frog's head and is behind the eyes over the approximate location of the optic tectum.

The inset in Figure 2 also shows the way in which leg flexions are assessed. The sensor from a Coulbourn Optical Densitometer (Model S71-40) is mounted on an adjustable arm and is positioned so that its sensor is directly opposite the lower segment of the frog's leg but is behind a rigidly mounted sheet of thin Plexiglas. The purpose of the Plexiglas is to prevent the frog from pressing against, and thereby moving, the sensor. As noted in Marsh, Hoffman, and Stitt (1979), with this kind of sensor, peak output (in volts) is directly proportional to the speed and amplitude of the overt reaction.

Yerkes employed a pendulum that closed successive switches to control the timing of his stimulus events. With our system, Coulbourn modules are used for this purpose. Moreover, whereas Yerkes employed bells, whistles, and percussive instruments to generate his acoustic signals, ours are generated by Coulbourn precision signal generators.

In their embodiment for our use, the apparatus and holder were constructed to accommodate a medium-sized leopard frog. Accordingly, the apparatus was approximately 9 in. tall and was fabricated of . 25 -in.-thick sheet aluminum. It should be noted, however, that the dimensions of both the apparatus and the holder could be made larger or smaller, depending on the species of frog to be tested.

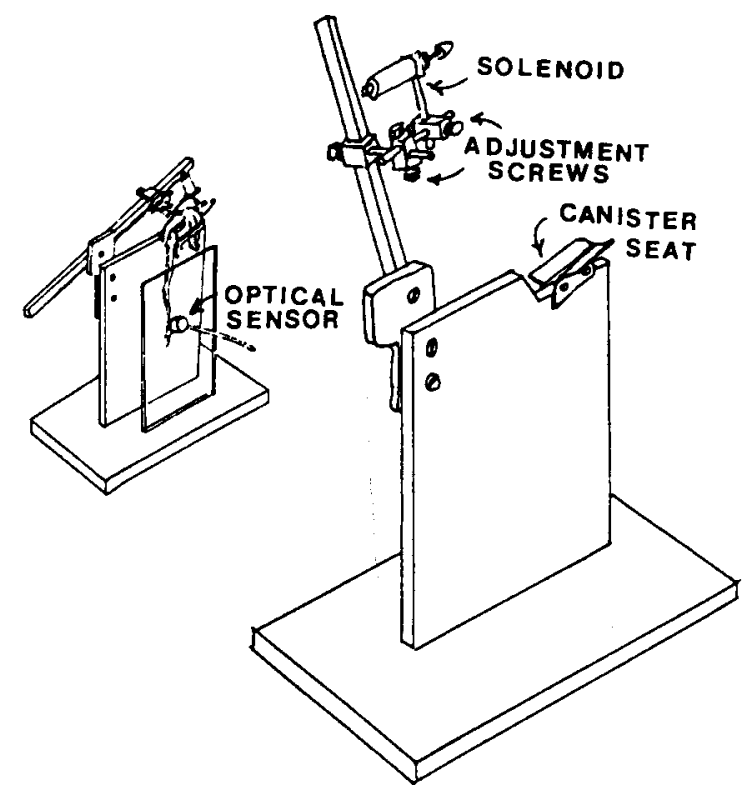

Figure 2. The body of this figure shows the apparatus into which the canister (containing a frog) is placed. The inset shows a frog in the apparatus with the tap delivery system in position for testing (see the text for details). 
In our effort to assess the efficacy of our system, each of 6 leopard frogs was placed in our apparatus and was exposed to an approximation to one of the procedures that Yerkes used. Yerkes had tested his frogs by presenting an acoustic signal prior to a tap on the frog's head and documenting the manner in which the amplitude of the leg flexion in response to the tap varied as a function of lead time. In our effort at replication, after being placed in the apparatus shown in Figure 2, a frog was exposed to a sequence of 50 taps in 10 blocks of five different conditions. As in Yerkes's original study, the tap was either presented alone or accompanied by an acoustic signal. However, whereas Yerkes's acoustic signal consisted of a percussive sound, ours consisted of a 40-msec 90-dB (re $20 \mu \mathrm{Pa}), 1-\mathrm{kHz}$ tone with a fast $(<0.1-\mathrm{msec})$ rise/fall time. As in Yerkes's study, whenever a tone was presented, it either occurred simultaneously with a tap or preceded the tap by one of several lead times. In our effort at replication, we elected to test three of the lead times that Yerkes had used-namely, 150, 450, and $900 \mathrm{msec}$. With our system, taps were generated by delivering a 40-msec pulse of $15-\mathrm{V}$ dc current to the solenoid. We arranged that a tap would occur once every $35 \pm 5 \mathrm{sec}$ and that all five of our stimulus conditions would occur within each block but that their order would change from block to block in a random fashion.

For each frog, we calculated the mean response amplitude for each of the five stimulus conditions that we tested. Figure 3 summarizes these calculations by showing the means (across subjects) of these means. The horizontal line indicates the mean response to the tap on the head when it was presented alone. The other four points indicate the means of the responses to the taps when they were accompanied by a tone. Since a one-way repeated measures analysis of variance of these data yielded $F(4,20)=15.27, p<$ .05 and a subsequent Neuman-Keuls analysis revealed significant differences between the tap-alone condition and the $0-$, as well as the 450 - and the $900-\mathrm{msec}$ conditions $(p<.05)$, it is clear that the effects summarized in Figure 3 are statistically reliable.

As noted earlier, Yerkes had observed that his frogs never directly reacted to his acoustic signals. We too found that our frogs never overtly reacted to our tones when they were presented alone. Yerkes had found, however, that his frogs' leg flexions to a tap on the head were enhanced by a simultaneously presented acoustic signal. We also found that when tone presentation was simultaneous with a tap, response amplitude was increased. Of equal importance, however, is that when the tone preceded the tap by either 450 or $900 \mathrm{msec}$, response amplitude was reduced, exactly as Yerkes had found.

In its overall form, the function that is shown in Figure 3 duplicates the functions that Yerkes plotted in his monograph. The only substantial difference is that for us, an acoustic signal with a $150-\mathrm{msec}$ lead time tended to inhibit the reflex elicited by the tap, whereas for Yerkes, an acoustic signal with a 150 -msec lead time tended to produce response enhancement. Since this kind of difference can readily arise from the differences in the kinds of sig-

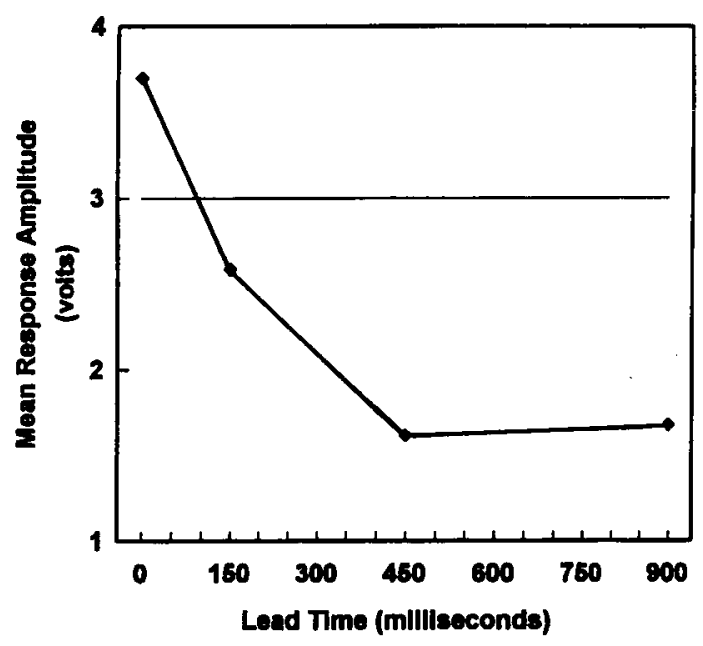

Figure 3. Mean amplitude of leg movement (across subjects) in response to a tap on the head. The horizontal line indicates the mean response amplitude when the tap was presented with no prior or concurrent acoustic signal. The other points indicate the mean response amplitude when the taps were preceded or accompanied by an acoustic signal.

nals that were used, it is concluded that the apparatus and procedures described here provide an effective way to examine prepulse effects in the frog. In doing so, they have the potential of extending Yerkes's leg flexion procedures to a wide range of new issues. For example, with appropriate recording devices, it would be possible to document the details of the manner in which various prepulses influence response latency. Moreover, given appropriate signal-generating devices, it would be possible to assess the effects of prepulses in a variety of modalities and to assess the effects of various combinations of prepulses from different as well as the same modality. Since both kinds of devices are readily available, there appears to be no substantial roadblock to such investigations.

\section{REFERENCES}

HoffMan, H. S., \& ISON, J. R. (1980). Reflex modification in the domain of startle: I. Some empirical findings and their implications for how the nervous system processes sensory input. Psychological Review, 87, 175-189.

Ison, J. R., \& Hoffman, H. S. (1983). Reflex modification in the domain of startle: II. The anomalous history of a robust and ubiquitous phenomenon. Psychological Bulletin, 94, 3-17.

MarSh, R. R., Hoffman, H. S., \& STITT, C. L. (1979). Eyeblink elicitation and measurement in the human infant. Behavior Research Methods \& Instrumentation, 11, 498-502.

Moss, C. F., \& Simmons, A. M. (1986). Frequency selectivity of hearing in the green treefrog, Hyla cinerea. Journal of Comparative Physiology $A, 159,257-266$.

SimmoNs, A. M. (1988). Selectivity for harmonic structure in complex sounds by the green treefrog, Hyla cinerea. Journal of Comparative Physiology A, 162, 397-403.

YERKES, R. M. (1905). The sense of hearing in frogs. Journal of Comparative Neurology \& Psychology, 15, 279-304.

(Manuscript received March 20, 1995; revision accepted for publication May 10, 1995.) 